\title{
Reactive Sulfur Species: Kinetics and Mechanism of the Equilibrium Between Cysteine Sulfenyl Thiocyanate and Cysteine Thiosulfinate Ester in Acidic Aqueous Solution
}

\author{
Kelemu Lemma and Michael T. Ashby* \\ Department of Chemistry and Biochemistry, University of Oklahoma, \\ 620 Parrington Oval, Norman, OK 73019
}

Submitted October 28, 2007

Revised December 202007

\section{SUPPORTING INFORMATION}

Derivation of the Rate Law of Eq 4:

$\begin{array}{ll}2\left\{\mathrm{CySSCN}+\mathrm{H}_{2} \mathrm{O}=\mathrm{CySOH}+\mathrm{SCN}^{-}+\mathrm{H}^{+}\right\} & \mathrm{k}_{1}, \mathrm{k}_{-1} \\ 2 \mathrm{CySOH} \rightarrow \mathrm{CyS}(=\mathrm{O}) \mathrm{SCy}+\mathrm{H}_{2} \mathrm{O} & \mathrm{k}_{2}\end{array}$

$2 \mathrm{CySSCN}+\mathrm{H}_{2} \mathrm{O} \rightarrow \mathrm{CyS}(=\mathrm{O}) \mathrm{SCy}+2 \mathrm{SCN}^{-}+2 \mathrm{H}^{+}$

$$
\text { Rate }=\frac{-1}{2} \times \frac{\mathrm{d}[\mathrm{CySSCN}]}{\mathrm{dt}}=\frac{\mathrm{d}[\mathrm{CyS}(=\mathrm{O}) \mathrm{SCy}]}{\mathrm{dt}}
$$

Assuming the second reaction is rate-limiting:

$$
\text { Rate }=\mathrm{k}_{2}[\mathrm{CySOH}]^{2}
$$

Applying the Steady-State Approximation:

$$
\frac{-\mathrm{d}[\mathrm{CySOH}]}{\mathrm{dt}}=2 \mathrm{k}_{2}[\mathrm{CySOH}]^{2}+\mathrm{k}_{-1}\left[\mathrm{H}^{+}\right]\left[\mathrm{SCN}^{-}\right][\mathrm{CySOH}]-\mathrm{k}_{1}[\mathrm{CySSCN}]=0
$$

$\left[\mathrm{H}^{+}\right]$and $\left[\mathrm{SCN}^{-}\right]$are constant:

$$
[\mathrm{CySOH}]_{\mathrm{ss}}=\frac{-\mathrm{k}_{-1}\left[\mathrm{H}^{+}\right]\left[\mathrm{SCN}^{-}\right] \pm \sqrt{\mathrm{k}_{-1}{ }^{2}\left[\mathrm{H}^{+}\right]^{2}\left[\mathrm{SCN}^{-}\right]^{2}+8 \mathrm{k}_{1} \mathrm{k}_{2}[\mathrm{CySSCN}]}}{4 \mathrm{k}_{2}}
$$


Let:

$$
\alpha=\frac{8 \mathrm{k}_{1} \mathrm{k}_{2}}{\mathrm{k}_{-1}{ }^{2}\left[\mathrm{H}^{+}\right]^{2}\left[\mathrm{SCN}^{-}\right]^{2}}
$$

Then:

$$
\begin{array}{r}
\left.[\mathrm{CySOH}]_{\mathrm{sS}}=\frac{\mathrm{k}_{-1}\left[\mathrm{H}^{+}\right]\left[\mathrm{SCN}^{-}\right]\left\{\sqrt{1+\frac{8 \mathrm{k}_{1} \mathrm{k}_{2}}{\mathrm{k}_{-1}{ }^{2}\left[\mathrm{H}^{+}\right]^{2}\left[\mathrm{SCN}^{-}\right]^{2}} \cdot[\mathrm{CySSCN}]}-1\right\}}{4 \mathrm{k}_{2}}\right\} \\
{[\mathrm{CySOH}]_{\mathrm{ss}}=\frac{\mathrm{k}_{-1}\left[\mathrm{H}^{+}\right]\left[\mathrm{SCN}^{-}\right]\{\sqrt{1+\alpha \cdot[\mathrm{CySSCN}]}-1\}}{4 \mathrm{k}_{2}} \cdot \frac{\sqrt{1+\alpha \cdot[\mathrm{CySSCN}]}+1}{\sqrt{1+\alpha \cdot[\mathrm{CySSCN}]}+1}} \\
{[\mathrm{CySOH}]_{\mathrm{ss}}=\frac{2 \mathrm{k}_{1}[\mathrm{CySSCN}]}{\mathrm{k}_{-1}\left[\mathrm{H}^{+}\right]\left[\mathrm{SCN}^{-}\right](\sqrt{1+\alpha[\mathrm{CySSCN}]}+1)}}
\end{array}
$$

Substituting the last eqn into the rate law and replacing $\alpha$ with its definition gives eqn 4 :

$$
\text { Rate }=\frac{-\mathrm{d}[\mathrm{CySSCN}]}{\mathrm{dt}}=2 \mathrm{k}_{2}\left\{\frac{2 \mathrm{k}_{1}[\mathrm{CySSCN}]}{\mathrm{k}_{-1}\left[\mathrm{H}^{+}\right]\left[\mathrm{SCN}^{-}\right](\sqrt{1+\alpha[\mathrm{CySSCN}]}+1)}\right\}^{2}
$$

\section{Derivation of the Rate Law of Eq 8:}

$\mathrm{CySSCN}+\mathrm{H}_{2} \mathrm{O}=\mathrm{CySOH}+\mathrm{SCN}^{-}+\mathrm{H}^{+} \quad \mathrm{k}_{1}, \mathrm{k}_{-1}$

$\mathrm{CySSCN}+\mathrm{CySOH} \rightarrow \mathrm{CyS}(=\mathrm{O}) \mathrm{SCy}+\mathrm{SCN}^{-}+\mathrm{H}^{+} \mathrm{k}_{2}$

$2 \mathrm{CySSCN}+\mathrm{H}_{2} \mathrm{O} \rightarrow \mathrm{CyS}(=\mathrm{O}) \mathrm{SCy}+2 \mathrm{SCN}^{-}+2 \mathrm{H}^{+}$

$$
\text { Rate }=\frac{-1}{2} \times \frac{\mathrm{d}[\mathrm{CySSCN}]}{\mathrm{dt}}=\frac{\mathrm{d}[\mathrm{CyS}(=\mathrm{O}) \text { SCy }]}{\mathrm{dt}}
$$

Assuming the second reaction is rate-limiting:

$$
\text { Rate }=k_{2}[\text { CySSCN][CySOH }]
$$

Apply the Steady-State Approximation:

$$
\frac{-\mathrm{d}[\mathrm{CySOH}]}{\mathrm{dt}}=\mathrm{k}_{2}[\mathrm{CySSCN}][\mathrm{CySOH}]+\mathrm{k}_{-1}\left[\mathrm{H}^{+}\right]\left[\mathrm{SCN}^{-}\right][\mathrm{CySOH}]-\mathrm{k}_{1}[\mathrm{CySSCN}]=0
$$

$\left[\mathrm{H}^{+}\right]$and $\left[\mathrm{SCN}^{-}\right]$are constant:

$$
[\mathrm{CySOH}]_{\mathrm{sS}}=\frac{\mathrm{k}_{1}[\mathrm{CySSCN}]}{\mathrm{k}_{2}[\mathrm{CySSCN}]+\mathrm{k}_{-1}\left[\mathrm{H}^{+}\right]\left[\mathrm{SCN}^{-}\right]}
$$

Substituting the last eqn into the rate law gives eqn 8: 


$$
\text { Rate }=\frac{\mathrm{k}_{1} \mathrm{k}_{2}[\text { CySSCN }]^{2}}{\mathrm{k}_{2}[\text { CySSCN }]+\mathrm{k}_{-1}\left[\mathrm{H}^{+}\right]\left[\mathrm{SCN}^{-}\right]}
$$

